\title{
Classes de comprimento e descrição histológica das gônadas de Diplodon ellipticus (Wagner, 1827) (Mollusca, Bivalvia, Hyriidae) em um lago artificial, Morretes, Paraná, Brasil
}

\author{
Ana Aparecida Nogueira Meyer * \\ Edinalva Oliveira \\ Tissiane Budach Teixeira \\ Jessica Aparecida Viesser \\ Universidade Positivo \\ Rua Prof. Pedro Viriato Parigot de Souza, 5300, CEP 81280-330, Curitiba - PR, Brasil \\ * Autor para correspondência \\ anameyer@onda.com.br
}

Submetido 20/11/2013

Aceito para publicação em 13/05/2014

\section{Resumo}

O objetivo deste estudo foi analisar a frequência por classes de comprimento e a organização histológica das gônadas de Diplodon ellipticus em um lago artificial localizado no município de Morretes-PR. Foram realizadas seis campanhas amostrais bimestrais no período de julho de 2009 a maio 2010, com captura de 150 espécimes. O comprimento total das conchas foi medido para determinação das frequências de classes de comprimento e foi realizada análise da presença de marsúpios nas brânquias. A determinação do sexo foi realizada por meio da análise histológica de secções transversais da massa visceral. Foram registradas oito classes de comprimento, com intervalo de $9 \mathrm{~mm}$, e a classe modal variou entre 50 a $59 \mathrm{~mm}$. A população é predominantemente dioica, com ocorrência de espécimes hermafroditas em baixa frequência. Marsúpios foram registrados em fêmeas (86\%) em todas as coletas amostrais e em dois exemplares hermafroditas. Análises histológicas demonstraram que a espermatogênese e ovogênese ocorrem ao longo do ano, indicando que D. ellipticus apresenta uma estratégia que proporciona uma reprodução contínua, com picos de liberação de gloquídios.

Palavras-chave: Ciclo reprodutivo; Marsúpio; Proporção sexual

\section{Abstract}

Length class and histological description of the gonads of Diplodon ellipticus (Wagner, 1827) (Mollusca, Bivalvia, Hyriidae) at an artificial lake, Morretes, Paraná, Brazil. This study aimed to analyze the frequency by length classes and the histological organization of gonads of Diplodon ellipticus at an artificial lake located in the town of Morretes, Paraná, Brazil. Six bimonthly sampling campaigns were conducted within the period from July 2009 to May 2010, with capture of 150 specimens. The total length of shells was measured for determining the frequencies of length classes and an analysis of the presence of marsupials in branchiae was performed. Sex determination was performed through histological analysis of cross sections of the visceral mass. We registered 8 length classes, with an interval of $9 \mathrm{~mm}$, and the modal class varied between 50 and $59 \mathrm{~mm}$. The population is predominantly dioecious, with occurrence of hermaphrodite specimens at low 
frequency. Marsupials were registered among females (86\%) in all sampling campaigns and in 2 hermaphrodite specimens. Histological analyses showed that spermatogenesis and oogenesis occur over the year, indicating that $D$. ellipticus has a strategy that provides continuous reproduction, with peaks of glochidia release.

Key words: Reproductive cycle; Marsupial; Sex ratio

\section{Introdução}

O declínio da população de moluscos límnicos da classe bivalve está relatado para todos os continentes e apresenta causas comuns, que incluem a degradação do ambiente associado a atividades antropogênicas, disseminação de espécies invasoras (DUDGEON; MORTON, 1983; VAUGHN; TAYLOR, 1999;ARAUJO; RAMOS, 2000; DOWNING et al., 2010) e características específicas deste grupo como, por exemplo, o longo ciclo de vida, crescimento lento e reduzida capacidade de dispersão (VAUGHN; TAYLOR, 1999).

Bivalves límnicos são considerados indicadores de qualidade ambiental devido ao relevante papel na filtração de partículas e algas da coluna d'água, excreção de nutrientes, depósito de pseudofezes, penetração de $\mathrm{O}_{2}$ no sedimento e estímulo ao metabolismo microbiano (VAUGHN; HAKENKAMP, 2001; VIDIGAL et al., 2005). Desta forma, a redução da população de bivalves pode contribuir para a diminuição da qualidade da água e ampliação da degradação ambiental de ambientes límnicos.

Apesar da representatividade destes organismos para os ecossistemas, no Brasil, onde ainda existe carência de inventários que possibilitem registros da ocorrência e distribuição para a maior parte dos estados, estima-se a existência de 117 espécies de bivalves límnicos (PEREIRA et al., 2013), dos quais 26 encontram-se na lista de ameaçados de extinção nas categorias vulneráveis, em perigo ou criticamente em perigo (MACHADO et al., 2008).

O gênero Diplodon, é considerado o mais primitivo Hyriidae da América do Sul, com ampla distribuição geográfica no continente, habitando todas as bacias hidrográficas, lagoas marginais, lagos e represas artificiais (BONETTO, 1962). A diversidade de ambientes que ocupa implica numa ampla variedade de formas, o que resulta em discussões sobre o número de espécies e sinonímias (MANSUR et al., 1987).
Segundo Pereira et al. (2013), na América do Sul, a espécie Diplodon ellipticus Wagner, 1827 está limitada a registros exclusivos para o Brasil e, de acordo com Simone (2006), a amplitude de distribuição estende-se do Espírito Santo ao Rio Grande do Sul, na bacia superior dos rios São Francisco e do Paraná. Apesar destes registros, as características da espécie são conhecidas apenas pelos estudos originais de descrição, baseados na morfologia das valvas e discussão sobre sinonímias (MANSUR; PEREIRA, 2006).

É senso comum à necessidade de ampliação de estudos anatômicos (MANSUR, 1973; AVELAR; CUNHA, 2009) e descrição de formas larvais do gênero que permitam o estabelecimento de características taxonômicas e diminuição das controvérsias apontadas para o gênero (MANSUR et al., 1987; MANSUR; SILVA, 1999). Contudo, a manutenção de populações das espécies do gênero igualmente necessita de abordagens que interpretem as características populacionais e do ciclo reprodutivo, e que contemplem informações relevantes para o planejamento de medidas que possibilitem reverter o quadro atual de ameaça às espécies de moluscos límnicos nativos.

No estado do Paraná, estudos relativos à distribuição e abundância de moluscos limnícos nativos são limitados e pontuais, principalmente em relação a grandes sistemas pluviais. Para a bacia hidrográfica do Iguaçu, Zanardini (1965) registrou a ocorrência de Diplodon expansus Kuster 1856, Diplodon multistriatus Lea 1831, Diplodon churruanus Orb 1835 e Diplodon supositus Simpson em rios de Curitiba. Curial e Lange (1974a; 1974b) analisaram a gametogênese, proporção sexual e relatos de hermafroditismo em Diplodon delontus expansus Kuster 1856, para o rio do Cerne, e Meyer et al. $(2010 ; 2013)$ registraram a ocorrência e analisaram a distribuição por frequência de classes de comprimento, proporção sexual e ocorrência sazonal de marsúpios em Diplodon expansus Kuster 1856, para o rio Piraquara, 
ambos localizados na Região Metropolitana de Curitiba. Porém, devido à falta de descrição detalhada de características taxonômicas e ausência de ilustrações, há necessidade de revisão das espécies do gênero Diplodon registradas para o estado do Paraná.

O objetivo do presente estudo foi analisar a frequência de classes de comprimento e a descrição histológica das gônadas, ao longo de um período de um ano, de Diplodon ellipticus ampliando o conhecimento sobre a biologia da espécie.

\section{Material e Métodos}

\section{Área de estudo}

A área de estudo está localizada no município de Morretes, em Área Especial de Interesse Turístico, a AEIT do Marumbi, criada pela Lei no 7.919 , de 22 de outubro de 1984 (ITCF, 1987) (Figura 1).
A vegetação desta área é composta principalmente pela Floresta Ombrófila Densa, além de áreas de refúgios vegetacionais e vegetação secundária. A classificação climática regional, de acordo com Köppen, corresponde ao tipo de clima subtropical úmido mesotérmico, com temperatura média anual variando entre 20,8 e $22^{\circ} \mathrm{C}$ (ITC, 1987).

O lago artificial $\left(25^{\circ} 27^{\prime} 236 \mathrm{~S}\right.$ e $\left.48^{\circ} 52^{\prime} 162 \mathrm{~W}\right)$ é abastecido por um afluente do rio Nhundiaquara, pertencente à Bacia Litorânea, constituída por um conjunto de sub-bacias, todas formadas por pequenos rios ou córregos (ITC, 1987). O lago apresenta tratamento paisagístico em seu contorno, com margens com gramíneas e vegetação exótica. O fundo apresenta substrato argiloso e uma lâmina de água variando entre $0,8 \mathrm{~m}$ nas margens e $1,5 \mathrm{~m}$ na região central. O lago destinado à piscicultura, sem fins comerciais, possui espécies de peixes característicos da fauna local, principalmente Siluriformes e Characiformes.

FIGURA 1: Mapa da localização geográfica da área de estudo.

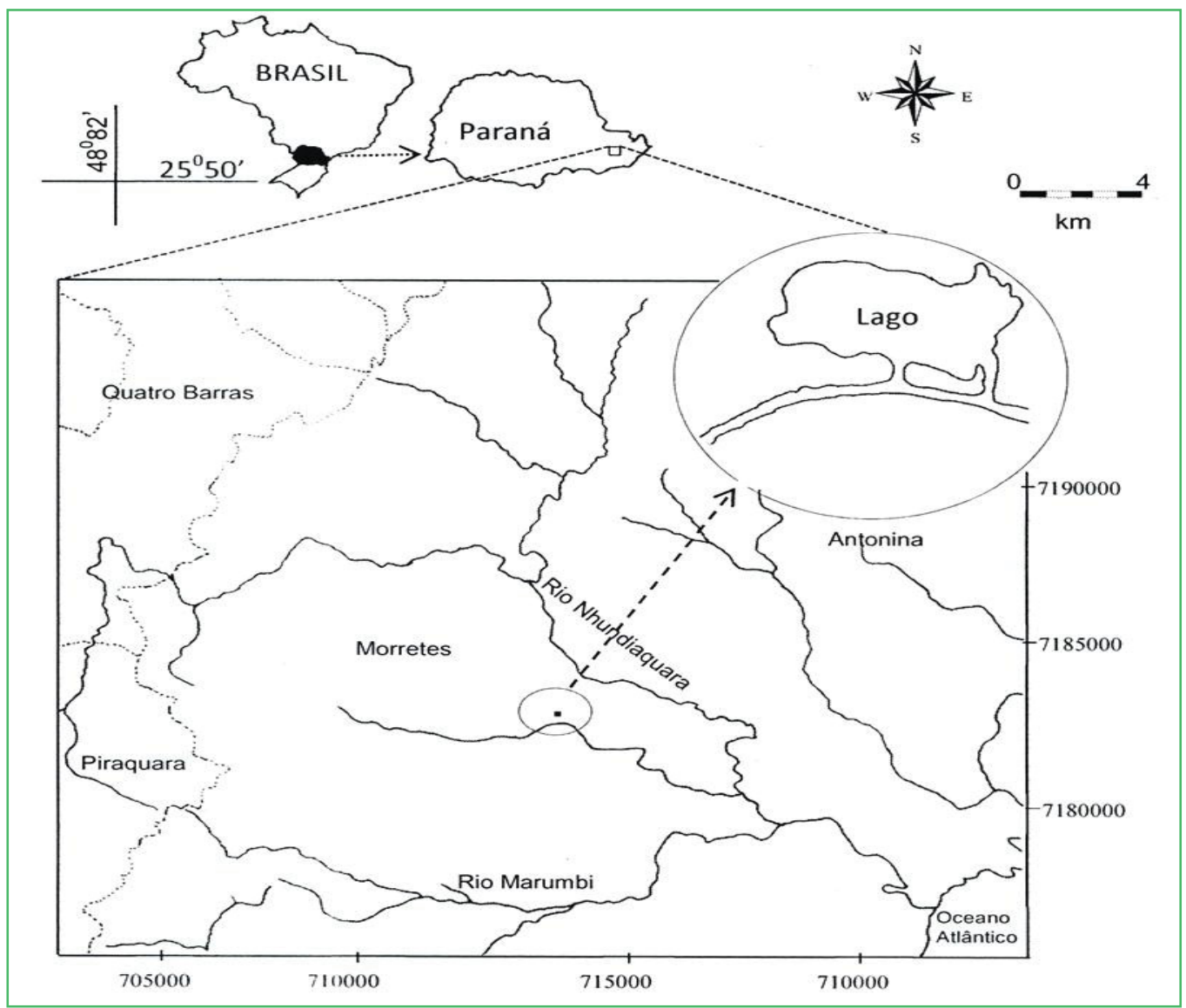


Dois canais artificiais de fundo lodoso, com folhiço, macrófitas e gramíneas nas margens e fundo arenoso na região central fazem a ligação do lago com um pequeno rio que é tributário de primeira ordem do rio Nhundiaquara. O canal de abastecimento apresenta largura de $1,6 \mathrm{~m} \mathrm{e}$ profundidade oscilando entre $0,2 \mathrm{~m}$ a $0,5 \mathrm{~m}$, enquanto que o canal de drenagem, que promove o retorno da água ao afluente do rio Nhundiaquara apresenta largura de $0,5 \mathrm{~m}$ e profundidade de $0,2 \mathrm{~m}$ (Figura 1).

\section{Atividades de campo e laboratório}

No lago, canais de abastecimento e drenagem foram realizadas seis campanhas amostrais bimestrais, no período entre julho de 2009 a maio 2010. Os dados abióticos foram mensurados em campo. A temperatura do ar e da água foram obtidas através de termômetro de mercúrio $\left({ }^{\circ} \mathrm{C}\right)$, a condutividade através de condutivimetria $(\mu \mathrm{S} / \mathrm{cm})$ e o $\mathrm{pH}$ com papel de tornassol. Para análise da relação entre as três variáveis abióticas estudadas (temperatura, condutividade e pH), foi usado um teste de correlação não paramétrico de Spearman, uma vez que, com uma amostra menor do que 30 elementos, as premissas de normalidade não podem ser atendidas (ZAR, 2009). As análises estatísticas foram efetuadas com o pacote estatístico Graphpad Prism e considerado um nível de significância de $5 \%(\alpha=0,05)$.

No lago, em cada campanha amostral, foram coletados por método de busca ativa e catação os 25 primeiros exemplares localizados no substrato. Nos canais de abastecimento e drenagem foram realizadas buscas ativas, com inserção de peneira, com abertura de malha de $1 \mathrm{~mm}$ junto ao substrato e macrófitas marginais. Os exemplares coletados foram submetidos a uma pré-abertura mecânica das valvas e fixados em formol 10\%. Em laboratório, cada exemplar recebeu identificação numérica e foi submetido à dissecação, com partes moles conservadas em álcool 70\% e as conchas arquivadas em via seca.

Exemplares testemunhos foram enviados ao Laboratório de Malacologia do Museu de Zoologia da USP e identificados por Luiz Ricardo Simone, como Diplodon ellipticus (Wagner, 1827) (Figura 2) e depositados na coleção de Mollusca, do Museu de Zoologia da Universidade de São Paulo (MZUSP), lote no 95186 .

Para a determinação da frequência de classes de comprimento, de cada exemplar da amostra coletada foi obtido o comprimento total da concha segundo Mansur et al. (1987), utilizando-se paquímetro de legibilidade $0,05 \mathrm{~mm}$. O intervalo de amplitude das classes de comprimento foi determinado a partir da aplicação da Regra de Sturgers.

Todos os exemplares foram examinados através de estereoscópio Leica ${ }^{\circledR}$ MZ12, para determinação da presença de marsúpios nas demibrânquias. Para análise da proporção sexual e da organização histológica da massa visceral foram obtidas, de 20 exemplares de cada coleta amostral, secções transversais da região central da massa visceral. As secções foram submetidas a processamento histológico de rotina, com desidratação, diafanização e inclusão em parafina. Cortes sequenciais de $5 \mu \mathrm{m}$ foram corados com corante de Hematoxilina e Eosina. A análise histológica foi realizada através de microscópio óptico Olympus $\mathrm{B} 400^{\circledR}$ e sistema de captação de imagens Image Pro Plus ${ }^{\circledR}$.

Os dados resultantes da sexagem foram utilizados para determinação das frequências de machos e fêmeas e a proporção sexual analisada através de teste de Quiquadrado. 
FIGURA 2: Exemplares de Diplodon ellipticus (Wagner, 1827) coletados entre julho de 2009 e maio de 2010, em lago artificial, Morretes, Paraná, Brasil. Vista externa, valva esquerda (A, E,). Vista externa, valva direita (B, F). Vista interna, valva esquerda (C, G). Vista interna, valva direita (D, H). Detalhe da vista interna, valva esquerda (I) e valva direita (J). Escala $1 \mathrm{~cm}$.

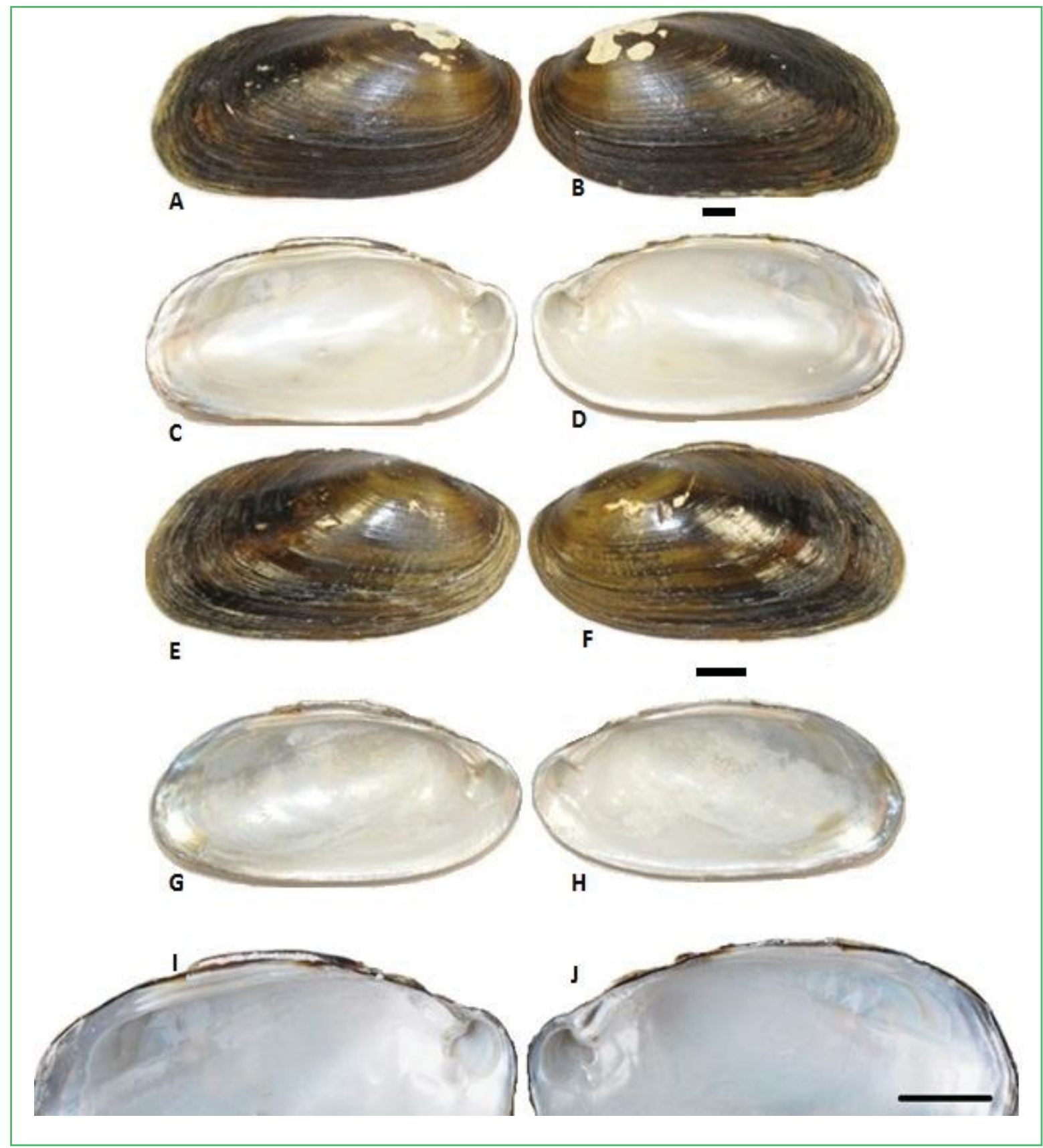




\section{Resultados}

Os dados abióticos em cada coleta amostral estão sumarizados na Tabela 1. As temperaturas da água do canal de abastecimento, do lago e do canal de drenagem se mantiveram próximas, exceto para os meses correspondentes ao verão, quando a diferença máxima de temperatura entre a água de abastecimento e do lago foi de $3{ }^{\circ} \mathrm{C}$. A condutividade da água foi maior nos meses com maior temperatura da água, com valores próximos entre o canal de abastecimento e o lago e superiores para o canal de drenagem. $\mathrm{O} \mathrm{pH}$ do canal de drenagem foi inferior ao observado para o lago e canal de abastecimento em todas as coletas amostrais.

As análises de correlação de Spearman, entre as variáveis abióticas, mostraram haver uma correlação entre a condutividade e a temperatura $(\mathrm{r}=0,68 ; \mathrm{p}=0,002)$, não observada entre a condutividade e o $\mathrm{pH}(\mathrm{r}=0,43$; $\mathrm{p}=0,078)$, e entre temperatura e $\mathrm{pH}(\mathrm{r}=0,08 ; \mathrm{p}=0,749)$.
No canal de abastecimento e canal de drenagem não foram registrados exemplares de $D$. ellipticus nas seis campanhas amostrais. Para a amostra total $(n=150)$ foram determinadas oito classes de comprimento, com amplitude de $9 \mathrm{~mm}$. O comprimento do menor exemplar foi de $14 \mathrm{~mm}$ e o maior de $86 \mathrm{~mm}$ e a classe modal com exemplares de 50 a $59 \mathrm{~mm}$ de comprimento $(\mathrm{n}=45)$.

A distribuição por frequência de comprimento, por campanha amostral, está representada na Figura 3 e os valores mínimo, médio, máximo e desvio padrão na Tabela 2. Foi registrada maior concentração de indivíduos nas classes de comprimento superior a $41 \mathrm{~mm}$ em todas as campanhas amostrais, exceto em março/10, quando a maior frequência foi de indivíduos pertencentes a classes de comprimento inferior a $50 \mathrm{~mm}$. Indivíduos inferiores a $14 \mathrm{~mm}$ estão ausentes durante o período de estudo e as classes de comprimento superiores a $77 \mathrm{~mm}$ apresentam baixa frequência.

TABELA 1: Parâmetros abióticos, do canal de abastecimento, lago artificial e canal de drenagem, obtidos no período de coletas, entre julho de 2009 e maio de 2010.

\begin{tabular}{ccccc}
\hline Parâmetros abióticos & Coleta & $\begin{array}{c}\text { Canal de } \\
\text { abastecimento }\end{array}$ & Lago & $\begin{array}{c}\text { Canal de } \\
\text { Drenagem }\end{array}$ \\
\hline \multirow{3}{*}{$\begin{array}{c}\text { Temperatura da água } \\
\left({ }^{\circ} \mathrm{C}\right)\end{array}$} & jul/09 & 18 & 17 & 19 \\
& set/09 & 20 & 20 & 20 \\
& nov/09 & 22 & 23 & 23 \\
& jan/10 & 22 & 25 & 24 \\
& mar/10 & 22 & 24 & 23 \\
\hline & maio/10 & 21 & 21 & 0,88 \\
Condutividade & jul/09 & 0,81 & 0,82 & 0,88 \\
$(\mu \mathrm{S} / \mathrm{cm})$ & $\mathrm{set} / 09$ & 0,87 & 0,82 & 1,19 \\
& $\mathrm{nov} / 09$ & 1,20 & 1,10 & 1,17 \\
& $\mathrm{jan} / 10$ & 1,20 & 1,09 & 1,21 \\
& $\mathrm{mar} / 10$ & 1,21 & 1,21 & 1,21 \\
\hline $\mathrm{maio} / 10$ & 1,22 & 1,03 & 5,5 \\
& $\mathrm{jul} / 09$ & 6,0 & 6,0 & 5,0 \\
$\mathrm{pH}$ & $\mathrm{set} / 09$ & 6,0 & 6,0 & 5,0 \\
& $\mathrm{nov} / 09$ & 6,0 & 6,0 & 5,0 \\
& $\mathrm{jan} / 10$ & 6,5 & 5,5 & 6,5 \\
& $\mathrm{mar} / 10$ & 7,0 & 6,0 & 5,5 \\
\hline
\end{tabular}


FIGURA 3: Distribuição por frequência de classes de comprimento (mm), por coleta amostral, de Diplodon ellipticus (Wagner, 1827) coletados em lago artificial, Morretes, Paraná, Brasil, entre julho de 2009 e maio de 2010.

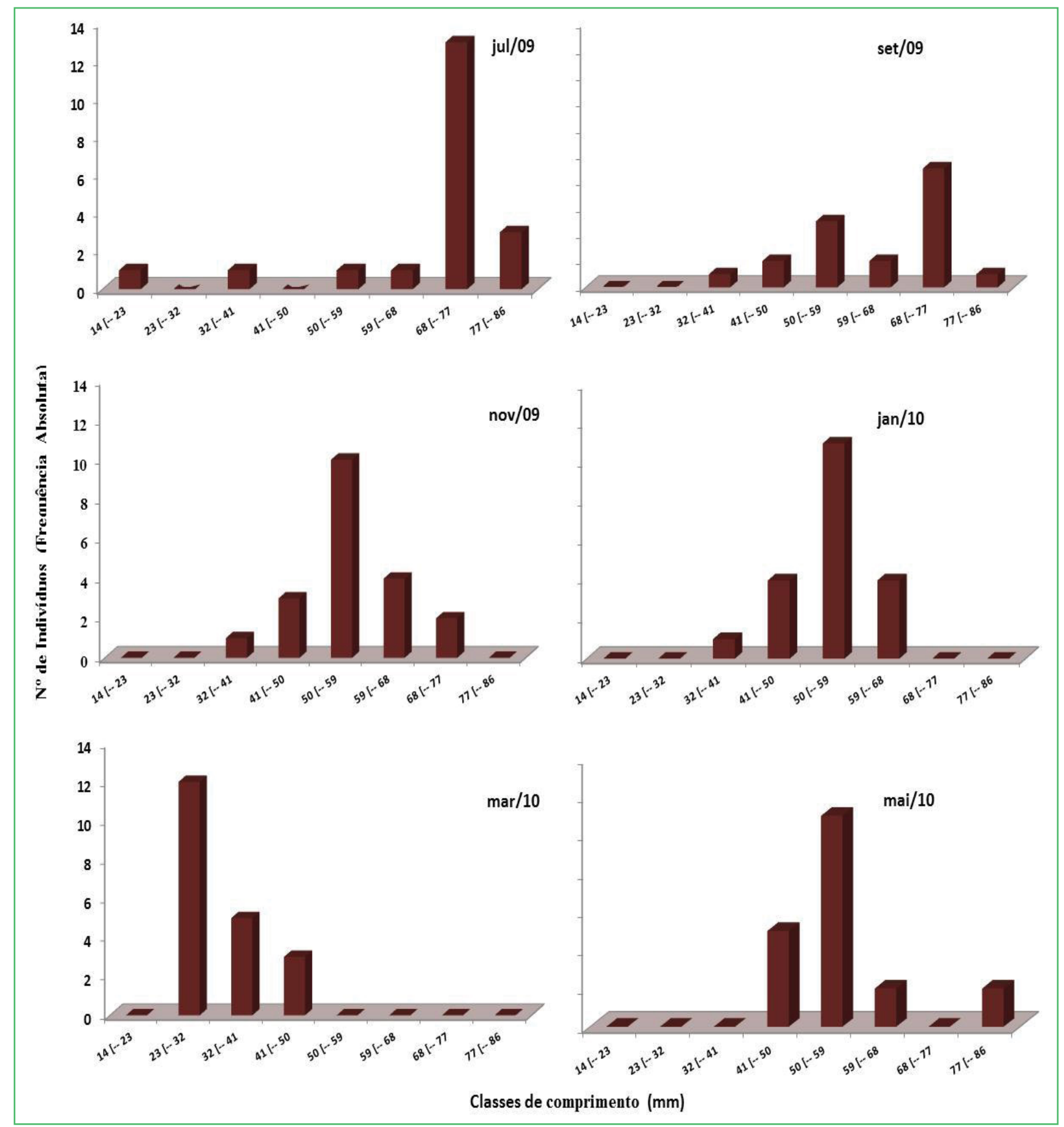


TABELA 2: Valores mínimo, médio, máximo e desvio padrão do comprimento das valvas de Diplodon elipticus (Wagner, 1827) coletados em lago artificial, Morretes, Paraná, Brasil, entre julho de 2009 e maio de 2010.

\begin{tabular}{ccccc}
\hline & Mínimo & Máximo & Média & $\begin{array}{c}\text { Desvio } \\
\text { Padrão }\end{array}$ \\
\hline jul/09 & 17 & 82 & 68,5 & $\pm 15,8$ \\
set/09 & 40 & 83 & 64,3 & $\pm 11,1$ \\
nov/09 & 40 & 71 & 53,8 & $\pm 6,9$ \\
jan/10 & 28 & 67 & 51,7 & $\pm 7,6$ \\
mar/10 & 14 & 77 & 56,3 & $\pm 11,6$ \\
maio/10 & 42 & 79 & 53,4 & $\pm 5,2$ \\
\hline
\end{tabular}

Através de técnicas histológicas foram identificados na amostra analisada, $(n=120)$ exemplares machos $(\mathrm{n}=61)$; exemplares fêmeas $(\mathrm{n}=52)$; exemplares hermafroditas $(n=4)$; exemplar imaturo $(n=1)$ e exemplares com alterações na massa gonadal que impossibilitaram a determinação do sexo $(n=2)$.

A análise de $\mathrm{X}^{2}$ da distribuição de frequência de machos e fêmeas, na amostra total ( $n=120)$, não evidenciou diferenças significativas, com a razão sexual observada de 1:1 $(\mathrm{p}<0,05)$. A frequência de machos, fêmeas e hermafroditas, por coleta amostral, está representada na Figura 4. Machos e fêmeas foram registrados em

FIGURA 4: Frequência de machos, fêmeas, imaturos e hermafroditas, por coleta amostral, de Diplodon ellipticus (Wagner, 1827) coletados em lago artificial, Morretes, Paraná, Brasil, entre julho de 2009 e maio de 2010.

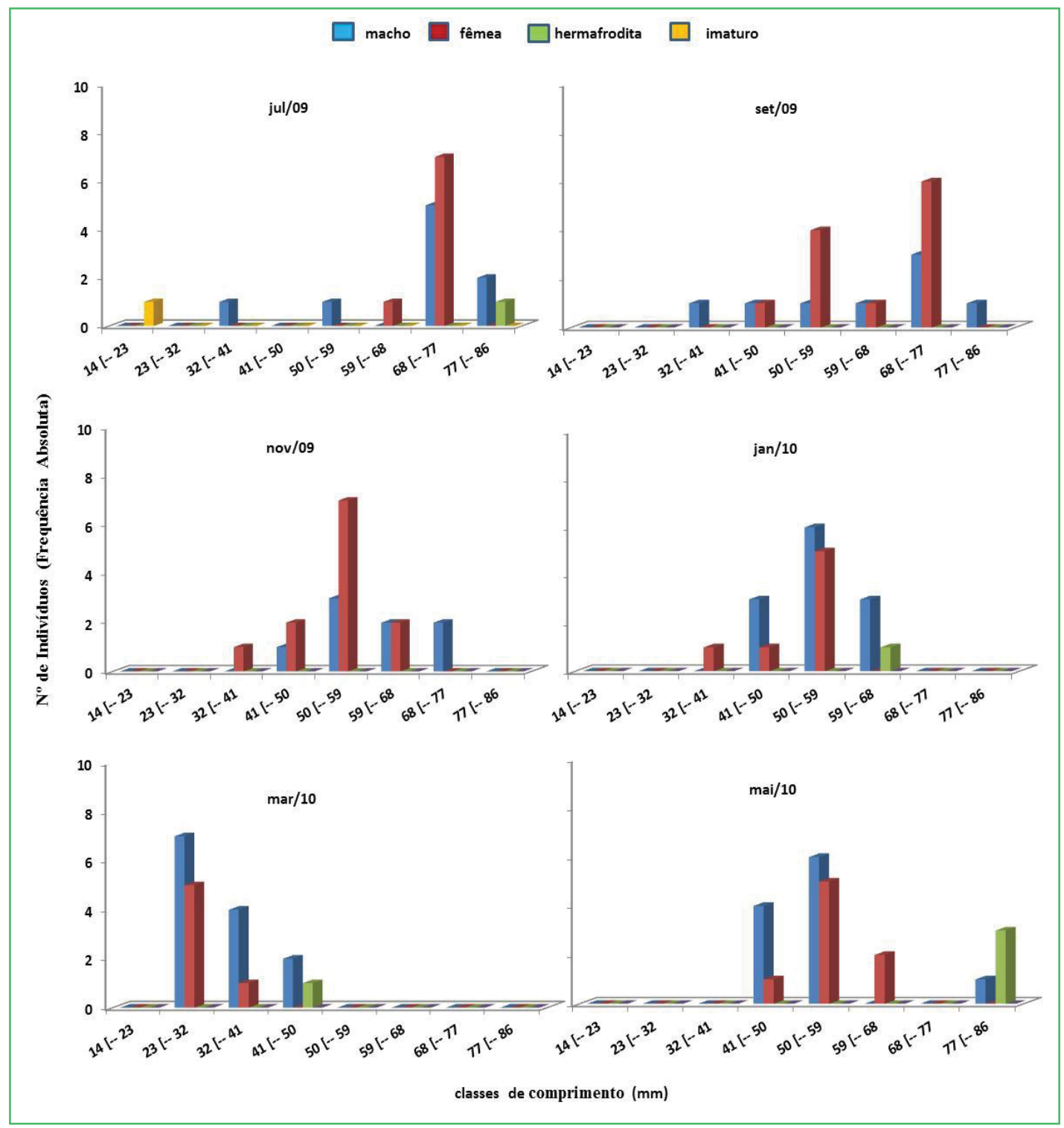


todas as campanhas amostrais e hermafroditas, em baixa frequência, foram registrados para as classes de comprimento acima de $50 \mathrm{~mm}$ em quatro campanhas amostrais. Um único indivíduo imaturo foi registrado na campanha amostral de julho/09. Ao longo do período amostral houve uma variação na distribuição de machos e fêmeas na classe modal, com fêmeas sendo mais numerosas em julho/09, setembro/09 e novembro/09 e machos em janeiro/10, março/10 e maio/10.

Em D. ellipticus, as gônadas estão localizadas na porção central da massa visceral, entre as dobras do intestino e estendendo-se até a região postero-dorsal do pé. Apresentam estrutura arborescente, semelhante a uma glândula ramificada tubulosa, o que resulta, devido à incidência de corte transversal, em folículos de aparência predominantemente esférica ou sacular.
As gônadas masculinas apresentam folículos bem delimitados e separados por escasso tecido conjuntivo (Figuras 5 e 6). Em todas as coletas amostrais, as gônadas de exemplares machos apresentam folículos com epitélio seminífero contendo células em diferentes estágios de diferenciação e formação de espermatozoides (Figuras 5 e 6). Espermatogonias e espermatócitos I formam grupamentos celulares nas regiões mais periféricas e espermátides e espermatozoides flagelados ocupam região mais próxima da luz do folículo (Figura 6). Em folículos que apresentam menor densidade de espermatozoides foi observada a formação de mórulas espermáticas (Figuras 7 e 8).

Os ductos espermáticos são ramificados, revestidos por tecido epitelial com células ciliadas, que apresentam citoplasma com fraca reação à eosina (Figura 5).

FIGURAS 5-8: Aspectos histológicos das gônadas masculinas de Diplodon ellipticus (Wagner, 1827) coletados em lago artificial, Morretes, Paraná, Brasil, entre julho de 2009 e maio de 2010. Folículo gonadal $(\diamond)$ e ductos espermáticos ( $\downarrow$ ); Folículo gonadal masculino com espermatogonias e espermatócitos I nas regiões periféricas $(\Delta)$; Espermatozoides na luz do folículo $(\nabla)$ e mórulas espermáticas $(\rightarrow)$.
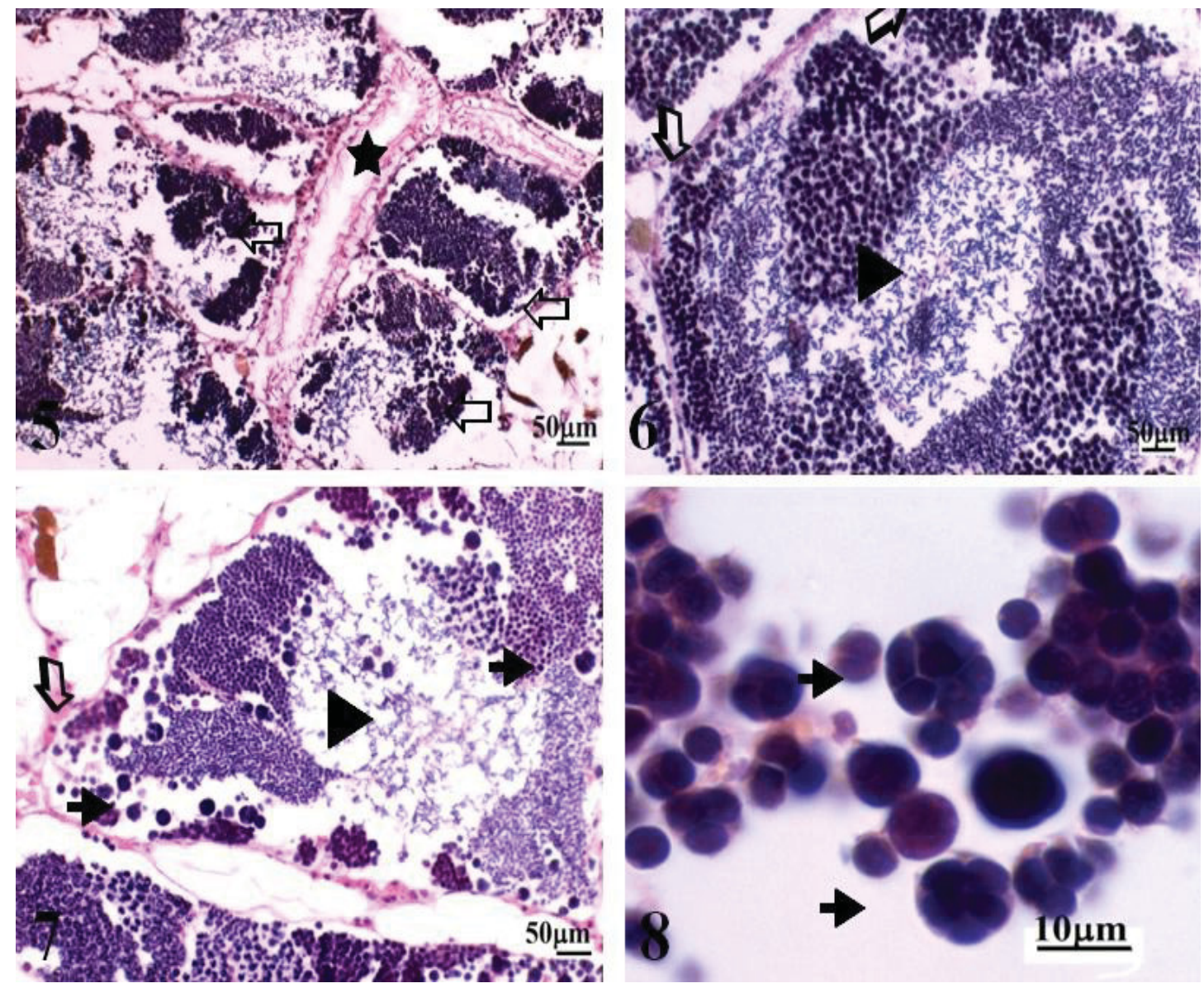
Em gônadas femininas, os folículos gonadais são separados por escasso tecido conjuntivo (Figura 9). Os ductos espermáticos são ramificados e revestidos por tecido epitelial com células ciliadas que apresentam citoplasma com fraca reação a eosina (Figura 10). Em todas as coletas amostrais foram identificados nos folículos gonadais, através das características morfológicas do núcleo, ovócitos em diferentes estágios de desenvolvimento. Ovócitos maduros, com núcleo apresentando cromatina dispersa, nucléolo evidente e citoplasma rico em vitelo são observados no lúmem do folículo (Figura 10). Ovócitos em crescimento apresentam núcleo com nucléolo evidente e permanecem ligados a células do epitélio de revestimento, através do peduncúlo citoplasmático (Figuras 11 e 12). As ovogônias são observadas entre as células da parede do folículo e podem ser identificadas pelo tamanho e citoplasma basófilo (Figura 12).

Em gonâdas de exemplares hermafroditas são observados, de forma predominante, folículos exclusivamente masculinos contendo todas as células da linhagem espermatogênica (Figura 13), e em menor quantidade, folículos exclusivamente femininos apresentando diferentes estágios de desenvolvimento (Figura 13). Nos folículos femininos, foi registrado para todos os exemplares hermafroditas da amostra, gônadas com folículos contendo ovócitos em desenvolvimento circundados por células da linhagem espermatogênica, grandes quantidades de mórulas espermáticas (Figuras 14 e 15) e folículos contendo massas eosinófilas, circundadas por células da linhagem espermatogenica e mórulas espermáticas (Figura16).

FIGURAS 9-12: Aspectos histológicos das gônadas femininas de Diplodon ellipticus (Wagner, 1827) coletados em lago artificial, Morretes, Paraná, Brasil, entre julho de 2009 e maio de 2010. Folículo gonadal (\); Folículos gonadais com ovócitos maduros ( ); Ductos espermáticos ( $\$ ); Ovócitos em desenvolvimento (४); ovócitos ligados à parede do folículo por pedúnculo citoplasmático $(\rightarrow)$; Parede do folículo com ovogônias $(\vec{\neg})$.
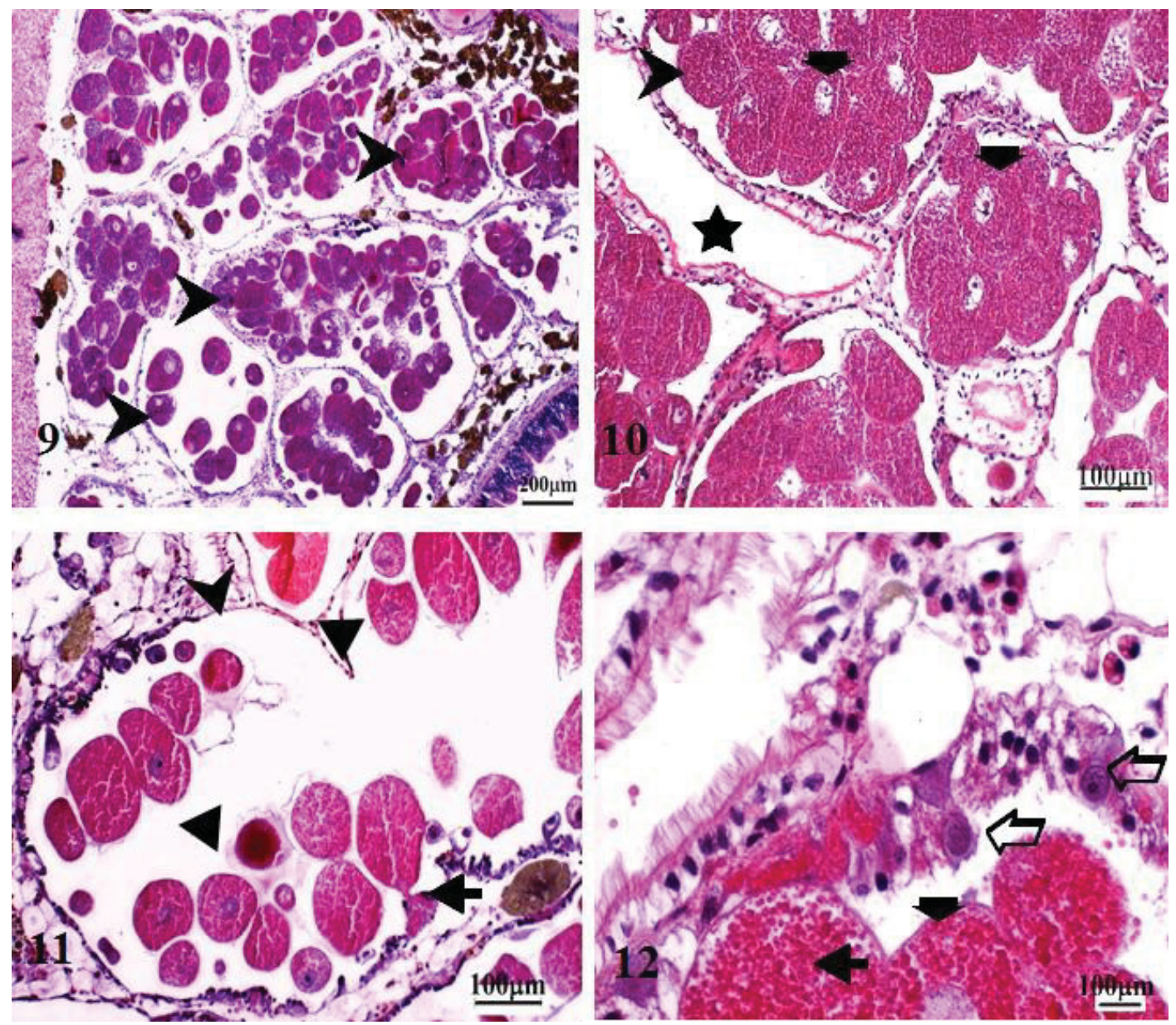
FIGURA 13-16: Aspectos histológicos das gônadas de exemplares hermafroditas de Diplodon ellipticus (Wagner, 1827) coletados em lago artificial, Morretes, Paraná, Brasil, entre julho de 2009 e maio de 2010. Folículo gonadal masculino ( $\triangleright$ ); Folículo gonadal feminino $(>)$; ovócitos em desenvolvimento $(\rightarrow)$; Ovócito preso por pedúnculo $(\rightarrow)$; Ovócito circundado por células da linhagem espermatogênica $(\mathbf{y})$; Mórulas espermáticas (ઐ); massa eosinófila circundada por células da linhagem espermatogênica $(\boldsymbol{*})$.
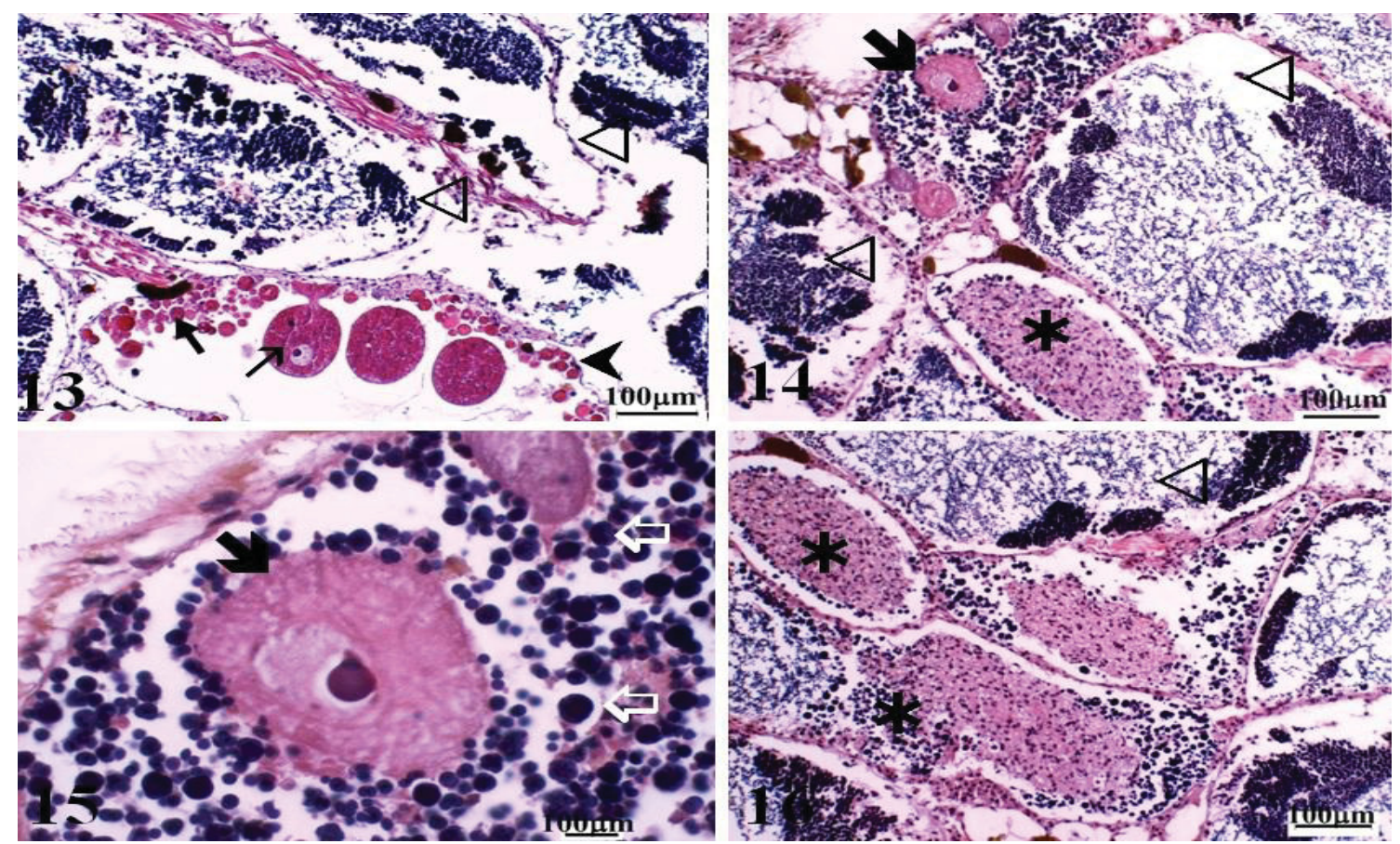

As brânquias de $D$. ellipticus são constituídas por marsúpios ocupam cerca de $70 \%$ da área linear das duas demibrânquias, uma interna e outra externa, que demibrânquias internas. apresentam a mesma forma e tamanho (Figura 17). Os

FIGURA 17: Massa visceral de Diplodon ellipticus (Wagner, 1827) coletado em lago artificial, Morretes, Paraná, Brasil, entre julho de

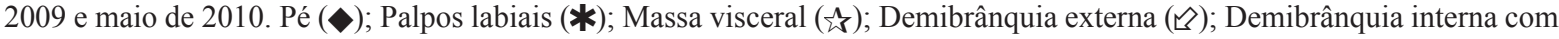
marsúpio (૯).
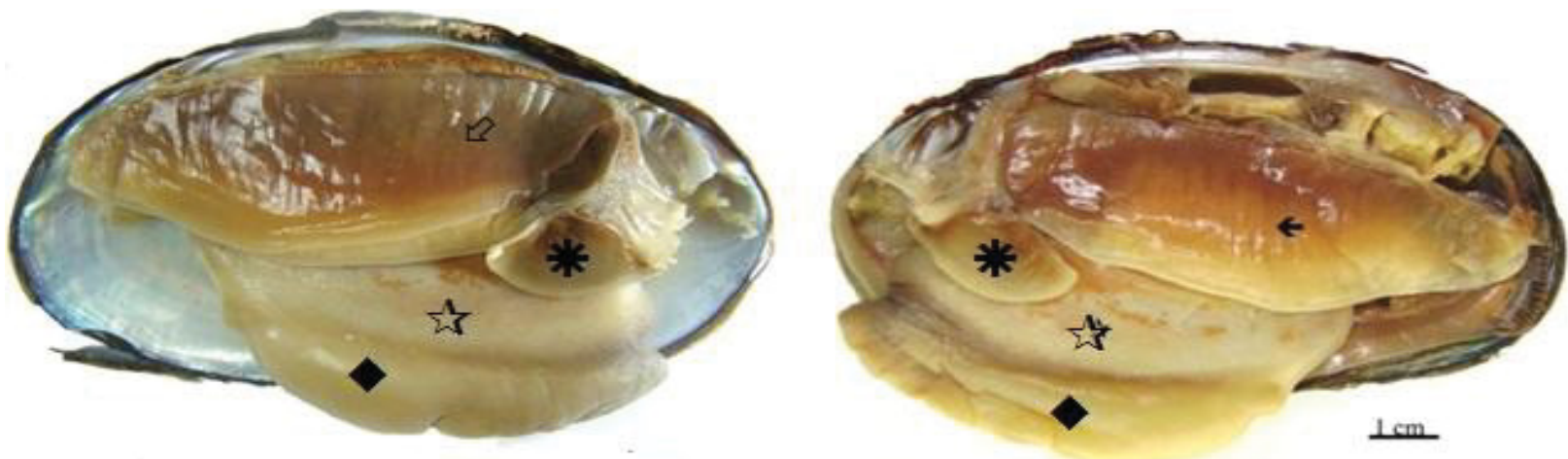
$\mathrm{Na}$ amostra total, das 51 fêmeas coletadas, $86 \%$ apresentaram marsúpios $(\mathrm{n}=44)$, e em indivíduos hermafroditas $(\mathrm{n}=4)$ foram observados marsúpios em dois exemplares. A ocorrência de fêmeas com marsúpios, por campanha amostral, está demonstrada na Figura 18. Fêmeas com marsúpio são registradas para todas as classes de comprimento superiores a $32 \mathrm{~mm}$ e são ausentes nas classes de comprimento superiores a $77 \mathrm{~mm}$. O maior número de fêmeas com marsúpio foi registrado para as classes de $50 \mathrm{~mm}$ a $59 \mathrm{~mm}$ em quatro campanhas amostrais.

FIGURA 18: Distribuição por frequência de classe de comprimento de fêmeas com marsúpio, por coleta amostral, de Diplodon ellipticus (Wagner, 1827) coletados em lago artificial, Morretes, Paraná, Brasil, entre julho de 2009 e maio de 2010.

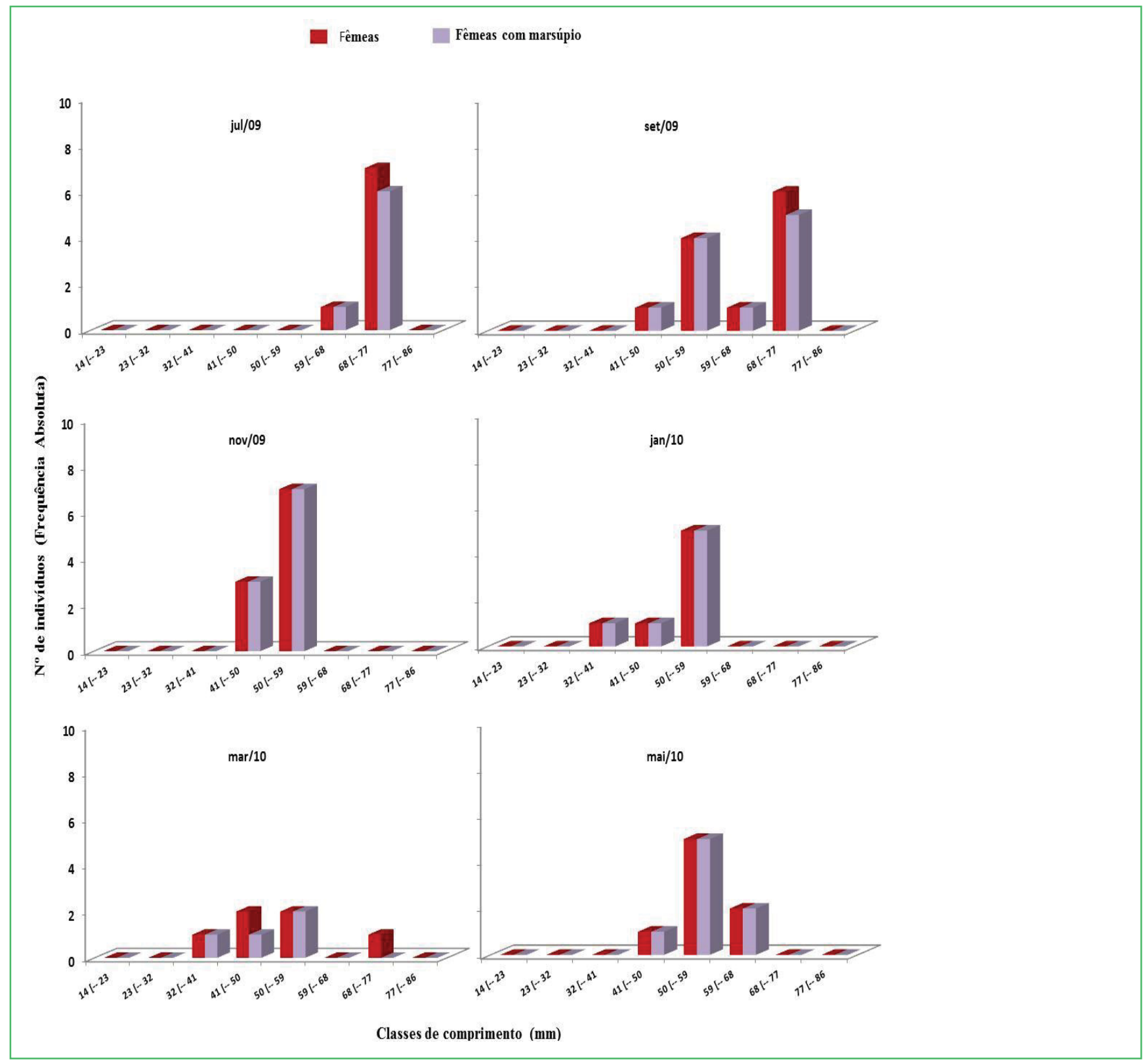




\section{Discussão}

As alterações e destruições de habitats por atividades antrópicas estão entre as ameaças comuns às populações de moluscos da Ordem Unionidae (BOX; MOSSA, 1999; PEREIRA et al., 2013). Contudo, no presente estudo, foi constatado que a formação de um lago artificial possibilitou a criação de um ambiente com condições para o estabelecimento da população de D. ellipticus.

Segundo comunicação oral dos proprietários da área, o lago foi formado a cerca de 20 anos, pelo método de escavação e abastecido desde sua origem, com água de tributário do rio Nhundiaquara. Como não há relato de utilização de nenhuma técnica de introdução de bivalves no lago, e em nenhuma das coletas amostrais foi registrada a presença de indivíduos jovens e adultos nos canais de abastecimento, é provável que a população tenha sido estabelecida a partir de larvas carreadas pela água que abastece o lago. Segundo Mansur et al. (1987), os Hyriidae possuem dois tipos de gloquídios, os que apresentam uma fase de desenvolvimento parasitário em peixes até a fase de pós-larva, e os que apresentam desenvolvimento completo até a fase de pós-larva no interior de marsúpios. Na literatura pesquisada, não existem registros de estudos da morfologia e tipo de gloquídios $D$. ellipticus, o que permitiria a compreensão da forma de introdução da espécie no lago artificial. Contudo, é possível inferir que as pós-larvas tenham sido liberadas por fêmeas a montante do desvio para abastecimento do lago e carreadas pela água, após sua liberação ou no período entre a fixação e liberação em peixes hospedeiros.

As diferenças entre o substrato do rio, que apresenta margens lodosas e leito central arenoso com rochas de diferentes granulometrias e o substrato lodo-argiloso do lago, não foi determinante para estabelecimento da população estudada. O gênero Diplodon foi registrado para substratos lodosos (ALVARENGA; RICCI, 1981) e substrato composto por areia fina (MANSUR, 1973). Meyer et al. (2010) relatam a ocorrência de D. expansus para trechos lodosos e arenosos do rio Piraquara, o que demonstra capacidade de adaptação, de espécies do gênero, aos dois tipos de substrato.
Os fatores abióticos, como a condutividade e a temperatura, são de grande importância para interpretação das condições de um ecossistema aquático, pois a condutividade indica a disponibilidade de macronutrientes (ESTEVES, 1988) e a temperatura está diretamente relacionada a picos reprodutivos e taxas de crescimento (PARADA et al., 1990; SEMENAS; BRUGNI, 2002). Como a condutividade é dependente da temperatura (ESTEVES, 1988), foi observada a correlação positiva entre as duas variáveis no lago em estudo, porém há necessidade de estudos complementares realizados em conjunto com outras variáveis, como turbidez e pluviosidade, para que seja possível avaliar a influência destes fatores abióticos sobre a população.

A ausência de fluxo de corrente, outro fator que contribui para as diferenças entre o lago e o rio, pode ter atuado de forma positiva para o sucesso no estabelecimento da população de D. ellipticus, pois contribui para a manutenção da distribuição agregada, característica do gênero, observada por Lara e Parada (1988), para D. chilensis Gray, 1828, Henry e Simão (1984), para D. delontus expansus, e Meyer et al. (2010), para $D$. expansus, o que segundo Amyot e Downing (1988), favorece o sucesso reprodutivo em época de desova. Além disso, a ausência de fluxo de corrente e a existência, no lago, de peixes em confinamento possibilitam o enriquecimento da água com nutrientes e sólidos dissolvidos, beneficiando os bivalves que são reconhecidamente eficientes filtradores de partículas em suspensão (VAUGHN; HAKENKAMP, 2001). Parada et al. (2008), em escala de laboratório, analisaram o potencial da espécie $D$. chilensis como depurador de águas residuais de tanques de piscicultura e registraram maior crescimento nos tanques com maior disponibilidade de partículas orgânicas.

Não foram registrados na literatura estudos populacionais de D. ellipticus para outras localidades, o que impossibilita comparações intraespecíficas, no entanto a amplitude de classes de comprimento entre 14 e $89 \mathrm{~mm}$ está dentro dos padrões observados para D. delontus expansus por Henry e Simão (1984) no reservatório do rio Pardo (SP) de 18-78 mm, e por Meyer et al. (2010) para $D$. expansus, que registraram amplitude 
de 17-65 mm para o rio Piraquara (PR). A ausência de indivíduos menores de $14 \mathrm{~mm}$, no presente estudo, pode ser atribuída ao método de amostragem por busca ativa e coleta manual, que inviabiliza a coleta de indivíduos jovens enterrados no substrato lodoso, também foi registrada, por Henry e Simão (1984) e Meyer et al. (2010; 2013).

A proporção macho/fêmea, na amostra total, não foi significativa, porém quando analisada a frequência de machos e fêmeas por coleta amostral se observa predomínio de fêmeas com marsúpio nos meses correspondentes a campanhas amostrais realizadas no inverno (jul/09) e na primavera (set/09 e nov/09), o que corresponde a picos de liberação de gloquídios, característicos do gênero, observados por Curial e Lange (1974b), para D. delontus expansus, Avelar e Mendonça (1998), para Diplodon rotundus gratus Wagner, 1827, e Meyer et al. (2010), para D. expansus.

A organização histológica das gônadas de fêmeas, machos e hermafroditas de D. ellipticus, com estrutura arborescente e ramificada, está de acordo com o descrito para o gênero por Curial e Lange (1974b), para D. delontus expansus, Peredo e Parada (1984), para Diplodon chilensis chilensis, Meyer et al. (2010), para D. expansus, e Oliveira et al. (2011), para Rhipidodontha burrougiana (Lea, 1834). As alterações gonadais observadas são semelhantes às registradas para $D$. expansus no rio Piraquara por Meyer et al. (2013), que relacionaram estas alterações a infestações parasitárias.

O menor exemplar coletado nas amostragens (14 $\mathrm{mm}$ ) apresentou raros folículos gonadais com epitélio germinativo indiferenciado, o que indica que a maturidade sexual ocorre em exemplares com comprimento de valva superior ao valor mínimo registrado.

A proporção sexual observada permite afirmar que a população estudada é tipicamente dioica, com exemplares hermafroditas apresentando baixa frequência, o que está de acordo com o observado para D. delontus expansus (CURIAL; LANGE, 1974a), D. chilensis (PEREDO; PARADA, 1984) e D. expansus (MEYER et al., 2010) e difere do observado para $D$. rotundus gratus (AVELAR; MENDONÇA, 1998) e $R$. burrougiana (OLIVEIRA et al., 2011), o que comprova a existência de variações na diferenciação sexual do gênero. De acordo com Coe (1943), $D$. ellipticus apresenta hermafroditismo funcional, pois possui produção simultânea de espermatozoides e ovócitos. Heard (1975) ampliou a classificação de Coe (1943) em hermafroditas $q$ e $\hat{\delta}$, utilizando como critério a predominância de folículos gonadais femininos ou masculinos e a presença de marsúpios em desenvolvimento. Nos exemplares hermafroditas de $D$. ellipticus analisados ocorreu um predomínio de folículos gonadais masculinos e presença de marsúpios com gloquídios em desenvolvimento na demibrânquia interna, o que difere dos padrões estabelecidos pelo autor.

A ocorrência de mórulas espermáticas nas gônadas masculinas e de hermafroditas de bivalves límnicos está bem relatada na literatura (HEARD, 1975; PEREDO; PARADA, 1984, JONES et al., 1986; AVELAR; MENDONÇA, 1998; MATOS et al., 1998; BEASLEY et al., 2000; CALLIL; MANSUR, 2007), todavia o significado funcional destas estruturas não é consenso entre os autores. Heard (1975) descreve as mórulas espermáticas, como resultado de espermatogênese atípica, pois originam espermatozoides com morfologia e tamanho diferentes da sequência espermatogênica típica, enquanto que Matos et al. (1998) postulam que a ocorrência de mórulas espermáticas é sazonal e que estas originam espermatozoides maduros típicos para a espécie.

$\mathrm{O}$ registro de espermatogênese e ovogênese e a ocorrência de marsúpios com larvas em desenvolvimento, ao longo de todo período amostral, indicam que $D$. ellipticus possui um ciclo reprodutivo contínuo, com dois picos de liberação de gloquídios.

\section{Agradecimentos}

Ao Programa de Iniciação Científica da Universidade Positivo. Ao Dr. Luiz Simone, curador da coleção de Malacologia do Museu da Universidade de São Paulo pela identificação e depósito de exemplares testemunhos de D. ellipticus. 


\section{Referências}

ALVARENGA, L. C.; RICCI, C. N. Bivalvia. In: HURLBERT, S. H; RODRIGUES, G.; SANTOS, N. D. (Ed.). Aquatic biota of Tropical South America. 2: Arthropoda. San Diego: San Diego State University, 1981. p. 208-217.

AMYOT, J. P.; DOWNING, J. A. Locomotion in Elliptio complanata (Mollusca: Unionidae) a reproductive function. Freshwater Biology, London, v. 39, n. 2, p. 351-358, 1988.

ARAUJO, R.; RAMOS, M. A. Status and conservation of the giant European freshwater Pearl mussel (Margaritifera auricularia) (Sppengler, 1793) (Bivalvia: Unionoidea). Biological Conservation, Boston, v. 96, p. 233-239, 2000.

AVELAR, W. E. P.; CUNHA, A. D. The anatomy and functional morphology of Diplodon rhombeus fontainianus (Orbigny, 1835) (Mollusca, Bivalvia, Hyriidae). Brazillian Journal Biology, São Carlos, v. 69, n. 4, p. 1153-1163, 2009.

AVELAR, W. E. P.; MENDONÇA, S. H. S. T. Aspectos of gametogenesis of Diplodon rotundus gratus (Wagner, 1872) (Bivalvia, Hyriidae) in Brazil. American Malacological Bulletin, Washington, v. 14, n. 2, p. 157-163, 1998.

BEASLEY, C. R.; TÚRY, E.; VALE, W. G.; TAGLIARO, C. H. Reproductive cycle, management and conservation of Paxydon syrmatophorus (Bivalvia: Hyriidae) from the Tocantins river, Brazil. Journal of Moluscan Studies, Oxford, v. 66, p. 393-402, 2000.

BONETTO, A. Investigaciones acerca de las formas larvales en el género Diplodon y su aplicación a los estúdios sistemáticos. Santa Fe: Dirección de Recursos Naturales, Ministério de Agricultura y Ganadería, 1962. 48 p.

BOX, J. B.; MOSSA, J. Sediment, land use, and freshwater mussels: prospects and problems. Journal North American Benthology Society, Glenview, v. 18, n. 1, p. 99-117, 1999.

CALLIL, C. T.; MANSUR, M. C. D, Gametogênese e dinâmica da reprodução de Anodontites trapesialis (Lamarck) (Unionoida; Mycetopodidae) no lago Baía do Poço, planície de inundação do rio Cuiabá, Mato Grosso, Brasil. Revista Brasileira de Zoologia, Curitiba, v. 24, n. 3, p. 825-840, 2007.

COE, W. R. Sexual differentiation in mollusks. Pelicypods. Quartely Review of Biology, Chicago, v. 18, p. 154-164, 1943.

CURIAL, O.; LANGE, R. R. Hermafroditismo em Diplodon delodontus expansus. Arquivos de Biologia e Tecnologia, Curitiba, v. 17, p. 109-110, 1974a.

CURIAL, O.; LANGE, R. R. Observações sobre a proporção de sexos em Diplodon delodontus expansus. Arquivos de Biologia e Tecnologia, Curitiba, v. 17, n. 2, p. 111-112, 1974b.

DOWNING, J. A.; VAN METER, P.; WOOLNOUGH, D. A. Suspects and evidence: a review of the causes of extirpation and decline in freshwater mussels. Animal Biodiversity and Conservation, Barcelona, v. 33, n. 2, p. 152-185, 2010.

DUDGEON, D.; MORTON, B. The population and sexual strategy of Anodonta woodiana (Bivalvia: Unionacea) in Plover Cove Reservoir, Hon Kong. Journal of Zoology, London, v. 201, p. 161-183, 1983.

ESTEVES, F. A. Fundamentos de Limnologia. 2 ed. Rio de Janeiro: Interciência, 1998. 602 p.

HEARD, W. H. Sexuality and other aspects of reproduction in Anadonta (Pelecypoda; Unionidae). Malacologia, Ann Arbor, v. 15, n. 1, p. 81-103, 1975.
HENRY, R.; SIMÃO, C. A. Evalluation of density and biomass of a bivalve population (Diplodon delontus expansus) (Küster, 1856) in a small tropical reservoir. Revista Hydrobiologia Tropical, San José, v. 17, n. 4, p. 309-318, 1984.

ITCF - INSTITUTO DE TERRAS E CARTOGRAFIA. Plano de gerenciamento da área especial de interesse turístico do Marumbi. Curitiba: ITCF, 1987. 105 p.

JONES, H. A.; SIMPSON, R. D.; HUMPHREY, R. D. The reproductive cycles and glochidia of freshwater mussels (Bivalvia: Hyriidae) of the Macleay River, northern South Wales, Australia. Malacologia, Ann Arbor, v. 27, p. 185-202, 1986.

LARA, G.; PARADA, E. Distribuicion espacial y densidad de Diplodon chilensis chilensis (Gray, 1828) en el Lago Villarrica $\left(39^{\circ} 18^{\prime} \mathrm{S} ; 7^{\circ} 05^{\prime} \mathrm{W}\right)$. Boletin de la Sociedad de Biologia de Concepción, Concepción, v. 59, p. 105-114, 1998.

MACHADO, A. B. M.; DRUMMOND, G. M.; PAGLIA, A. P. (Ed.). Livro vermelho da fauna brasileira ameaçada de extinção. Vol. 1. Brasília: Ministério do Meio Ambiente, 2008. 510 p.

MANSUR, M. C. D. Morfologia do sistema digestivo das espécies do gênero Diplodon SPIX, 1827 do rio Guaíba, Rio Grande do Sul (Unionacea-Hyriidae). Iheringia, Série Zoológica, Porto Alegre, v. 43, p. 75-90, 1973.

MANSUR, M. C. D.; PEREIRA, D. Bivalves límnicos da bacia do rio dos Sinos, Rio Grande do Sul, Brasil (Bivalvia, Unionoida, Veneroida e Mytiloida). Revista Brasileira de Zoologia, Curitiba, v. 23, n. 4, p. 1123-1147, 2006.

MANSUR, M. C. D.; SCHUlZ, C.; GARCES, L. M. M. P. Moluscos bivalves de água doce: identificação dos gêneros do sul e leste do Brasil. Acta Biologica Leopoldensia, São Leopoldo, v. 9, n. 2, p. 181-202, 1987.

MANSUR, M. C. D.; SILVA, M. G. O. Description of glochidia of five species of freshwater mussels (Hyriidae: Unionidae) from South America. Malacologia, Ann Arbor, v. 41, n. 2, p. 475-483, 1999.

MATOS, E.; CORRAL, L.; AZEVEDO, C. Fine structure of spermiogenesis with special reference to the spermatid morulae of the freshwater mussel Prisodon alatus (Bivalvia, Unionoidea). Journal of Morphology, Malden, v. 238, p. 63-70, 1998.

MEYER, A. A. N.; MARTIN, J.; OLIVEIRA, E. Ocorrência e caracterização histológica de marsúpios de Diplodon expansus (Küster, 1856) (Mollusca, Bivalvia, Hyriidae) no rio Piraquara, Paraná Brasil. Biotemas, Florianópolis, v. 26, n. 1, p. 97-108, 2013. MEYER, A. A. N.; OLIVEIRA, E.; MARTIN, J. Classes de comprimento e proporção sexual em Diplodon expansus (Mollusca, Bivalvia, Hyriidae) no rio Piraquara, Paraná, Brasil. Iheringia, Série Zoologica, Porto Alegre, v. 100, p. 329-335, n. 4, 2010.

OLIVEIRA, E.; MEYER, A. A. N.; MARTIN, J. K.; GNOATTO, L.; JANUÁRIO, T. A. P. Ocorrência e características da população de Rhipidodonta burroughiana (Lea, 1834) (Molusca, Bivalvia, Hyriidae) na Lagoa Dourada, Parque Estadual de Vila Velha, Paraná, Brasil. In: CARPÁNEZZI, O. T. B.; CAMPOS, J. B. (Org.). Coletânea de pesquisas: Parques Estaduais de Vila Velha, Cerrado e Guartelá. Vol. 1. Curitiba: Instituto Ambiental do Paraná, 2011. p. 187-194.

PARADA, E.; PEREDO, S.; CÁRDENAS, S.; VALDEBENITO, I.; PEREDO, M. Diplodon chilensis Gray, 1828 (Bivalvia: Hyriidae) um potential depurador de aguas residuales de piscicultura 
de salmonidos de aguas continentales: um estúdio a escala de laboratório. Gayana, Concepción, v. 72, n. 1, p. 68-78, 2008.

PARADA, E.; PEREDO, S.; GALLARDO, C. Tácticas reproductivas y dinâmica poblacional de Diplodon chilensis (Gray, 1828) (Bivalvia; Hyrridae). Revista Chilena de Historia Natural, Santiago, v. 63, p. 23-35, 1990.

PEREDO, S.; PARADA, E. Gonadal organization and gametogenesis in the fresh-water mussel Diplodon chilensis chilensis (Mollusca: Bivalvia). The Veliger, Santa Bárbara, v. 27, n. 2, p. 126-133, 1984.

PEREIRA, D.; MANSUR, M. C. D; OLIVEIRA, A. S.; PIMPÃO, D. M.; CALLIL, C. T.; ITUARTE, C.; PARADA, E.; PEREDO, S.; DARRIGRAN, G.; SCARABINO, F.; CLAVIJO, C.; LARA, G.; MIYAHIRA, I. C.; RODRIGUEZ, M. T. R.; LASSO, C. Bivalve distribution in hydrographic regions in South America: historical overview and conservation. Hydrobiologia, Brussels, On line first, doi: 0.1007/s10750-013-1639-x, 2013. Disponivel em: <http:/ link.springer.com/search?query=bivalve+distribution\&searchwithin $=$ Journal \& facet-publication-title $=\% 22$ Hydrobiologia $\% 22>$. Acesso em: 29 outubro 2013.
SEMENAS, L.; BRUGNI, N. Características póblacionales y ciclo de vida de Diplodon chilensis (D'Orbigny, 1835) (Hyriidae, Bivalvia) em el lago Gutiérrez (Patagonia, Argentina). Ecologia Austral, Buenos Aires, n. 12, p. 29-40, 2002.

SIMONE, L. R. L. Land and freshwater mollusks of Brazil. São Paulo: EGB, FAPESP, 2006. 390 p.

VAUGHN, C. C.; HAKENKAMP, C. C. The functional role of burrowing bivalves in freshwater ecosystems. Freshwater Biology, London, v. 46, p. 1431-1446, 2001.

VAUGHN, C. C.; TAYLOR, C. M. Impoundments and the decline of freshwater mussels: a case study of an extinction gradient. Conservation Biology, San Francisco, v. 13, n. 4, p. 912-920, 1999.

VIDIGAL, T. H. D. A.; MARQUES, M. M. G. S. M.; LIMA, H. P.; BARBOSA, F. A. R. Gastrópodes e bivalves límnicos do trecho médio da bacia do Rio Doce, Minas Gerais, Brasil. Lundiana, Belo Horizonte, v. 6 (suplemento), p. 67-76, 2005.

ZANARDINI, I. F. Nota sobre Diplodon e Anodontites (Mollusca - Pelecypoda) de rios de Curitiba (Paraná). Boletim do Instituto de Defesa do Patrimônio Natural, Curitiba, v. 6, p. 1-11, 1965.

ZAR, J. H. 2009. Biostatistical analysis. 5. ed. London: Prentice Hall. 950 p. 